\title{
Acalculous cholecystitis in malaria: Complication or accomplice?
}

\author{
Sokwala A., MBChB, Kamraan H., MBBS, FCPS and Abdallah A., MBChB, H Dip Surg (SA), FRCS, \\ Aga Khan University Hospital, Nairobi, Department of Surgery, P.O. Box 30270-00100, Nairobi, Kenya \\ Email: abdulkarim.abdallah@aku.edu
}

\section{Summary}

Acalculous cholecystitis is an acute necro inflammatory disease of the gallbladder with multifactorial pathogenesis. Acute acalculous cholecystitis (AAC) typically occurs in critically ill or injured patients but has also been associated with various infectious agents. We report here a case of AAC in a patient with Plasmodium falciparum malaria.

\section{Case Report}

A 64-year-old Kenyan male was admitted with a one week history of fever, headache, nausea and vomiting. The symptoms appeared two weeks after he had returned from Uganda. On admission, his temperature was $38^{\circ} \mathrm{C}$, pulse 102 beats/min, and his blood pressure was 126/88 $\mathrm{mm} \mathrm{Hg}$. Physical examination was essentially normal apart from mild dehydration.

Laboratory studies showed a normal cell count with white blood cell (WBC) count of $9.1 \times 10^{9} / \mathrm{L}$ with $74 \%$ neutrophils, $15 \%$ lymphocytes, $8 \%$ monocytes and $3 \%$ eosinophils, haemoglobin level of $13.5 \mathrm{~g} / \mathrm{dl}$ and platelet count of $94 \times 10^{9} / \mathrm{L}$. Examination of blood thin smear revealed infestation with ring trophozoites, typical of Plasmodium falciparum with $2 \%$ of erythrocytes being parasitized. Treatment with intramuscular b-Artemether (Paluther) was started. On the second day the patient developed abdominal pain and continued to have fever $\left(39^{\circ} \mathrm{C}\right)$, nausea and vomiting. Physical examination demonstrated increased tenderness and inspiratory arrest on subcostal palpation of the right upper quadrant (positive Murphy's sign). Liver function tests showed an elevated SGOT at $134 \mathrm{iu} / \mathrm{L}(\mathrm{N}<37$ $\mathrm{IU} / \mathrm{L}$ ), alanine aminotransferase (ALT) at 136 $\mathrm{iu} / \mathrm{L}(\mathrm{N}<40 \mathrm{IU} / \mathrm{L})$ in the presence of a total bilirubin level of $82 \mathrm{umol} / \mathrm{L}(\mathrm{N}<17 \mu \mathrm{mol} / \mathrm{L})$, and a direct bilirubin level of $42 \mu \mathrm{mol} / \mathrm{L}(\mathrm{N}<4$ $\mu \mathrm{mol} / \mathrm{L})$. GGT was also elevated at $207 \mathrm{IU} / \mathrm{L}$ (N 11-50 iu/L ) and alkaline phosphatase was 180 $\mathrm{iu} / \mathrm{L}$ (N 98-270 IU/L). Liver ultrasound showed a thickened gall bladder wall measuring $7 \mathrm{~mm}$ which was suggestive of cholecystitis (Figure 1). No calculus was demonstrated. Ultrasonic murphy's was positive. The liver parenchyma was homogenous and the common bile duct was not dilated. An impression of ACC was made and intravenous cefuroxime and metronidazole were started. Blood cultures had no growth after 48 hours. The fever and the pain subsided after 48 hours. Artemether dose was completed and two doses of Mefloquine 500mg was given eight hours apart to complete the combination therapy guideline of treating malaria. The patient was well and discharged after five days. He was followed up in the outpatient clinic where his repeat liver ultrasound after six months was normal with the gall bladder wall thickness of $3.1 \mathrm{~mm}$ (Figure 2). His liver function tests had also normalized. 
Figure 1: Initial ultrasound of the gallbladder wall thickness $7 \mathrm{~mm}$

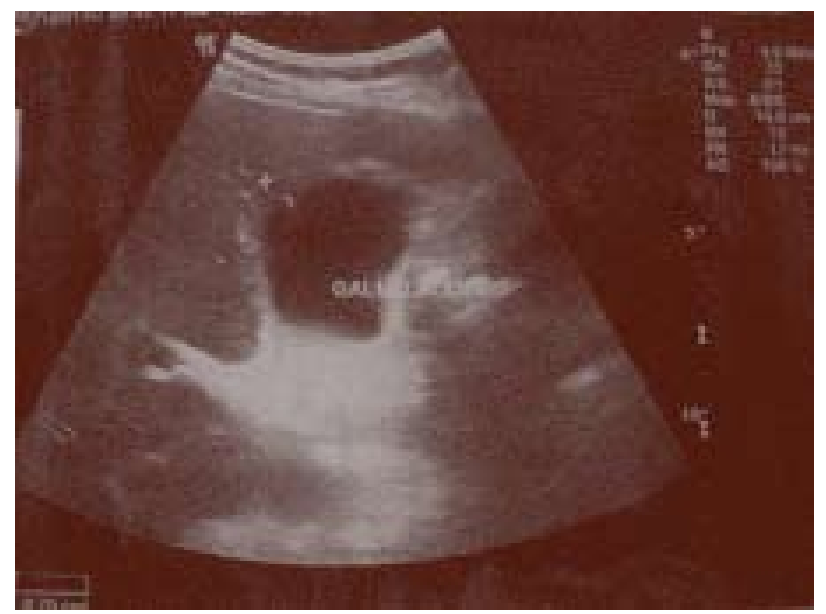

\section{Discussion}

According to our knowledge this is the first well documented case of acute acalculous cholecystitis secondary to malaria from East Africa despite being an endemic area for malaria. Very few cases have been reported worldwide describing association of acute acalculous cholecystitis with malarial infection in adults $(1,2)$. Most of the cases are from India. The first association between malaria and cholecytitis was described by Pizzillo in 1950 as pseudocholecystitis and pseudoappendicitis (3). AAC has been described in patients in association with a number of conditions like trauma, burns, abdominal surgery, prolonged parenteral nutrition and infections. Common infections associated with AAC include dengue fever, salmonellosis, brucellosis, leptospirosis, staphylococcal infection, isospora sp, cyclospora $\mathrm{sp}$, leishmania $\mathrm{sp}$ and other systemic illnesses $(4,5)$.

Symptoms of AAC include fever, right upper quadrant pain, nausea and vomiting, most of which are common in malaria as well. Tenderness to palpation or a mass in the right hypochondrium are often noted on clinical examination. The diagnosis can be confirmed on abdominal ultrasound which shows gall bladder distension, thickening of the gall
Figure 2: Repeat ultrasound of gallbladder, wall thickness $3.1 \mathrm{~mm}$

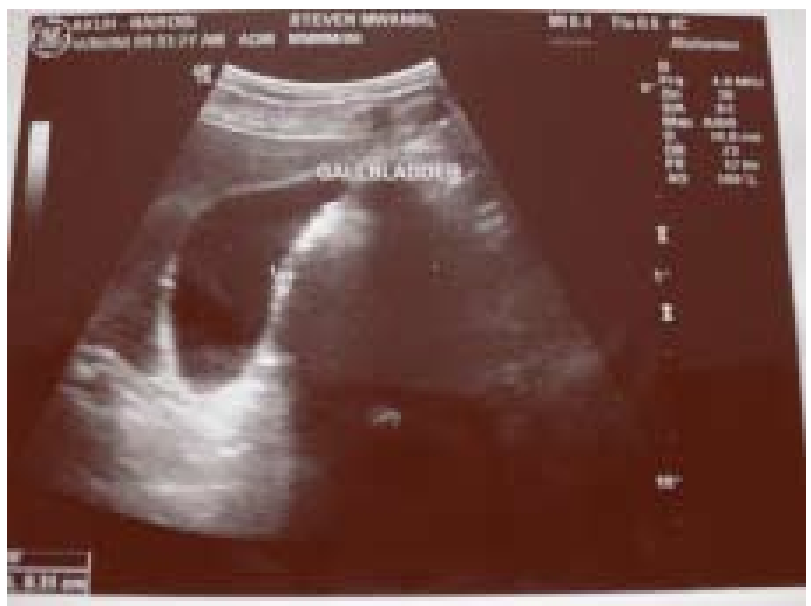

bladder wall $(>3.5 \mathrm{~mm})$, sludge in gall bladder or pericholecystic fluid (6).

The exact cause of AAC in systemic infection and illness is not known. Gall bladder wall ischemia and reperfusion injury, and the effects of proinflammatory mediators, appear to be important (7). Bile stasis due to fasting state in critical illnesses has been implicated to be a contributing factor. Microcirculatory obstruction in the gall bladder due to the phenomenon of rosetting and cytoadherence of $P$. falciparum-infected red blood cells might have further contribution to the ischemia of gall bladder and thus to the pathogenesis of AAC in patients with falciparum malaria (8). Thereafter secondary infection by enteric flora can take place. Derangement of liver enzymes, synthetic and cannalicular whilst not a typical feature of acute acalculus cholerystitis, is not surprising in the acute setup and usually normalise once the inflammation settles down.

AAC is classically associated with a high rate of life threatening complications (gangrene and perforation), and mainstay of treatment has been cholecystectomy or percutaneous cholecystostomy. However, uncomplicated AAC frequently resolves with the treatment of the underlying infective condition as was the case with our patient. Complicated and nonresolving cases require cholecystostomy or cholecystectomy $(8,9)$. 


\section{Conclusion}

Acute acalculous cholecystitis is a rare complication of $P$ falciparum malaria. It can either be caused by malaria parasite itself as postulated above or as a secondary bacterial infection in addition to malaria infection. Gastrointestinal symptoms, such as nausea, vomiting, and diarrhea, are common to both Plasmodium falciparum malaria and AAC, Attention to upper quadrant signs should raise suspicion for AAC.

\section{References}

1. Maggi P., Coppola S.L., Lamargese V., et al. Acute acalculous cholecystitis associated with co-infection by Plasmodium falciparum and Plasmodium vivax. J. Infect. 2002; 44: 136-137.

2. Yombi J.C., Meuris C.M., Van Gompel A.M., et al. Acalculous cholecystitis in a patient with Plasmodium falciparum infection: A case report and literature review. J. Travel Med. 2006; 13: 178-180.
3. Pizzilo G. and Serio F. Acute abdominal syndromes in malaria: Pseudo-cholecystitis and pseudoappendicitis. Riv. Malariol. 1950; 29(5): 315-320.

4. Shapiro M.J., Luchtefeld W.B., Kurzweil S., et al. Acute acalculous cholecystitis in the critically ill. Amer. Surg. 1994; 60: 335.

5. McChesney J.A., Northup P.G. and Bickston S.J. Acute acalculous cholecystitis associated with systemic sepsis and visceral arterial hypoperfusion: A case series and review of pathophysiology. Dig. Dis. Sci. 2003; 48: 1960.

6. Imamoglu M., Sarihan H., Sari A., et al. Acute acalculous cholecystitis in children: Diagnosis and treatment. J. Ped. Surg. 2002; 37: 36-39.

7. Barie P.S. and Eachempati S.R. Acute acalculous cholecystitis. Curr. Gastroenterol. Rep. 2003; 5: 302-309.

8. Saha A., Batra P., Vilhekar K.Y., et al. Acute acalculous cholecystitis in a child with Plasmodium falciparum malaria. Ann. Trop. Paediatr. 2005; 25: 141-142.

9. Sachdev H.P.S., Sharma S., Khandpur S.C., et al. Sonography in the diagnosis and management of acute acalculous cholecystitis. Indian Pediatr. 1987; 24: 379-384 